\title{
Relationship between shear energy input and sedimentation properties of exopolysaccharide-producing Streptococcus thermophilus strains
}

\author{
Florian Häffele $^{\mathrm{a}, *}$, Susann Mende ${ }^{\mathrm{b}}$, Doris Jaros ${ }^{\mathrm{b}}$, Harald Rohm ${ }^{\mathrm{b}}$, Hermann Nirschl ${ }^{\mathrm{a}}$ \\ a Institute of Mechanical Process Engineering and Mechanics (MVM), Karlsruhe Institute of Technology (KIT), Straße am Forum 8, 76131 Karlsruhe, Germany \\ ${ }^{\mathrm{b}}$ Chair of Food Engineering, Institute of Natural Materials Technology, Technische Universität Dresden (TUD), Bergstraße 120, 01062 Dresden, Germany
}

Keywords:

Analytical centrifugation

Sedimentation behavior

Starter cultures

Exopolysaccharides

Shear forces

\begin{abstract}
A B S T R A C T
Besides fermentation, the production of bacterial starter cultures includes another crucial step, namely, the separation of the bacteria cells. This separation is most commonly carried out with disc stack separators and needs to be adjusted to the respective strain to obtain a high cell recovery rate. Exopolysaccharides (EPS) produced by several starter cultures, however, have a large negative impact on the separation properties of the cells. These EPS can be divided into cell-bound capsular EPS or free EPS that are released into the surrounding fermentation medium. To improve the separation step, shear forces were applied after fermentation with a gear ring disperser to simulate the impact of a homogenizer and the influence on the separation properties of six Streptococcus thermophilus strains was examined. In case of capsular EPS, the sedimentation velocity of the bacteria increased due to shearing off the capsular EPS layer. Shearing media with free EPS resulted in a viscosity decrease and, hence, in a higher sedimentation velocity, as was determined using a disc centrifuge and a LUMiSizer. Sediment compression as measured with the LUMiSizer was also affected by the shearing step. The results of this study suggest that a defined shear treatment of EPS producing bacterial starter cultures leads to improved separation properties and, hence, higher bacteria yields. We assume that both EPS types affect separation efficiency of the bacteria cells, free EPS because of increased media viscosity and capsular EPS because they act like a friction pad.
\end{abstract}

\section{Introduction}

The importance of starter cultures that are able to produce exopo lysaccharides (EPS), for example several species of lactic acid bacteria (LAB), has significantly increased in recent years. In case of fermented dairy products, EPS influence product viscosity and mouthfeel. In case of yogurt and related products, LAB utilize lactose and produce lactic acid. A consequence of the decreasing $\mathrm{pH}$ is that the main milk protein fraction, the caseins, change their state and aggregate, such that the viscosity increases drastically $[1,2]$. This effect is enhanced when the respective bacteria are able to produce EPS during fermentation. EPS are long chained polysaccharides that consist of either identical monosaccharides (homo polysaccharide) or different monosaccharides (hetero polysaccharide), such as glucose, galactose, or rhamnose [3,4]. Due to their high water binding capacity, EPS have a large influence on the viscosity of the entire system. For instance some EPS are very ropy and able to interact with each other or the cells in the medium leading to an increase in viscosity. Examples of EPS that are, in isolated form, used in the food and pharmaceutical industries are dextran, a homo polysaccharide from Leuconostoc mesenteroides, or xanthan, a hetero polysaccharide from Xanthomonas campestris [5]. Furthermore, it is important to know that EPS produced by LAB are either attached to the cell wall (therefore denoted as capsular EPS) or released into the sur rounding medium (free EPS). The capability of producing either EPS type is strain dependent, a combination of both EPS types is also pos sible [6].

Industrial production of starter cultures consists of two main process steps, fermentation under specific conditions, such as $\mathrm{pH}$, temperature, oxygen availability, and nutrient content, and subsequent separation of the bacteria from the fermentation broth, which is usually done with disc stack separators. Here, the influence of EPS on the viscosity of the fermentation broth makes the separation step difficult. Another com plicating factor is that different starter cultures are usually produced in the same manufacturing line, which is why separation conditions should be adjusted to the respective strain. Both EPS characteristics and type (free, capsular or both) have a specific influence on the viscosity of the fermentation broth and, hence, on the sedimentation properties of the bacteria. Empirical observations indicate that the application of a

\footnotetext{
* Corresponding author.

E-mail address: florian.haeffele@kit.edu (F. Häffele).
} 
Table 1

Capsular EPS production and average cell chain length of unsheared Streptococcus thermophilus strains.

\begin{tabular}{lll}
\hline Strain & Presence of capsular EPS & Cocci per cell chain \\
\hline ST-C & + & $1-5$ \\
ST-D & & $2-19$ \\
ST-E & & $1-4$ \\
ST-G & + & $1-8$ \\
ST-H & + & $1-6$ \\
ST-I & + & $1-5$ \\
\hline
\end{tabular}

homogenization step before cell separation results in improved sedi mentation properties of the cultures, but also that this improvement depends on the EPS type produced. The aim of this study is to sys tematically investigate the effect of a homogenization step on the se paration properties of EPS producing lactic acid bacteria.

\section{Materials and methods}

\subsection{Materials}

Six different Streptococcus thermophilus (ST) strains dispersed in fermentation broth were investigated. The samples were delivered as frozen pellets by Chr. Hansen A/S (Horsholm, Denmark), stored at $-20^{\circ} \mathrm{C}$ and, before use, defrosted overnight in a refrigerator. The strains differed in average cell chain length and in their ability to produce capsular EPS (Table 1).

\subsection{Shear treatment}

A T25 Ultra Turrax (UT) gear ring disperser (IKA Werke GmbH \& Co. KG, Staufen, Germany) was used for specific shearing of the ST containing fermentation media. $30 \mathrm{ml}$ of the sample were filled into a test tube $\left(\mathrm{h}=200 \mathrm{~mm}, \mathrm{~d}_{\mathrm{i}}=28 \mathrm{~mm}\right)$ and sheared at either 5,000 , $11,000,19,000$, or $24,000 \mathrm{rpm}$. At each stirring speed, samples were taken (about $0.3 \mathrm{ml}$ with a syringe) after $10 \mathrm{~s}, 20 \mathrm{~s}, 30 \mathrm{~s}, 60 \mathrm{~s}$, and $120 \mathrm{~s}$ of shearing.

\subsection{Sedimentation velocity analysis (LUMiSizer)}

The sedimentation velocity distribution of unsheared and sheared samples was measured with an optical analytical centrifuge (LUMiSizer, LUM GmbH, Berlin, Germany). This centrifuge allows the simultaneous measurement of twelve samples using a rectangular polycarbonate cuvette $(2 \times 8 \mathrm{~mm}$ base area) for each sample. The cuvettes are inserted into the LUMiSizer in horizontal position and irradiated lengthwise with parallel NIR light. The NIR light transmitted through the cuvette is detected with a CCD sensor. The resulting time and place resolved transmission profiles are then evaluated by using the STEP technology (Space and Time resolved Extinction Profiles) and provide information on the sedimentation properties of the samples. More information re garding the working principle of the LUMiSizer and the STEP tech nology can be found in [7 9].

The measurements were carried out at a rotational speed of $3600 \mathrm{rpm}$, which corresponds to a mean relative centrifugal force (rcf) of $1720 \mathrm{~g}$ (mean distance from rotor was $120 \mathrm{~mm}$ ). Approximately $0.3 \mathrm{ml}$ diluted sample (ratio 1:2 with physiological $\mathrm{NaCl}$ solution) were filled into each cuvette. The sedimentation velocity distribution was calculated with the SEPVIEW program using the constant position method. For this, the mean transmission (averaged over $\pm 0.5 \mathrm{~mm}$ relating to the radial position) at three radial positions close to the bottom of the cells $(123,125$, and $127 \mathrm{~mm})$ was used. For each sample, two measurements were carried out.

\subsection{Sedimentation velocity analysis (Disc Centrifuge)}

The sedimentation velocity distribution of the unsheared and sheared samples was also measured with a DC24000 disc centrifuge (CPS Instruments Europe, Oosterhout, Netherlands). Here, the sample is injected centrally into a stirring vertical disc and deposited through a density gradient with known viscosity and density. After a certain time, the particles pass a laser beam at the outer edge of the pellucid disc and the absorption time spectrum of the sample is measured. By calibrating the centrifuge with a calibration standard with known particle size and sedimentation behavior, the absorption time spectrum of the sample can be transferred to the sedimentation velocity distribution. More detailed information regarding the working principle of the disc cen trifuge can be found in [10].

The stirring speed was set to $5850 \mathrm{rpm}$, which corresponds to $\mathrm{rcf}=1720 \mathrm{~g}$. The measuring range was set from $400 \mathrm{~nm}$ to $5 \mu \mathrm{m}$ and, because of the highly concentrated sample, a dilution with physiolo gical $\mathrm{NaCl}$ solution (ratio 1:20) was necessary. As a density gradient, a combination of a 3 and a $7 \%(w / w)$ sucrose solution was used. Therefore $1.6 \mathrm{ml}$ of the $7 \%(\mathrm{w} / \mathrm{w})$ sucrose solution was injected into the disc followed by a combination of $1.4 \mathrm{ml}$ of the $7 \%(\mathrm{w} / \mathrm{w})$ and $0.2 \mathrm{ml}$ of the $3 \%(\mathrm{w} / \mathrm{w})$ sucrose solution. The next step included $1.2 \mathrm{ml}(7 \%)$ and $0.4 \mathrm{ml}(3 \%)$ and so forth. At the end solely $1.6 \mathrm{ml}$ of the $3 \%(\mathrm{w} / \mathrm{w})$ sucrose solution was injected. The density gradient was prevented from evaporating by adding $0.5 \mathrm{ml}$ dodecane, followed by the recommended 15 min equilibration time. The PVC calibration standard had an average particle size of $0.483 \mu \mathrm{m}$ (PVC Calibration Standard: Lot 149 CPS Instruments Europe, Oosterhout, Netherlands) and was dispersed in deionized water. The injected sample (and calibration) volume was $0.1 \mathrm{ml}$. One calibration run was used as the basis for eight measure ments.

\subsection{Assessment of energy input}

The energy input from the shear treatment with the Ultra Turrax was approximated according to [11,12]. The Reynolds number Re ( ) near the rotor was calculated by

$R e=\frac{n \cdot d_{R}^{2} \cdot \varphi_{l}}{\eta_{l}}$

where $\mathrm{n}(1 / \mathrm{s})$ is the rotational speed, $\mathrm{d}_{\mathrm{R}}(\mathrm{m})$ stirrer diameter, $\rho_{1}\left(\mathrm{~kg} / \mathrm{m}^{3}\right)$ fluid density, and $\eta_{1}(\mathrm{~kg} / \mathrm{m} . \mathrm{s})$ apparent viscosity of the cell containing fermentation broth. As stated by [12,13], the flow pattern generated by the Ultra Turrax is similar to that caused by a disc stirrer. Zlokarnik [11] characterized many different types of stirrers, including the disc stirrer, and plotted a typical dependency of Newton number on the Reynolds number for each stirrer. With this characteristic, it is possible to assign a Newton number Ne ( ) to the calculated Reynolds number. Consequently, the input performance of the Ultra Turrax is calculated by:

$P=N e \cdot n^{3} \cdot d_{R}^{5} \cdot \varphi_{l}$

Finally, the volume specific energy input $\mathrm{E}_{\mathrm{V}}\left(\mathrm{J} / \mathrm{m}^{3}\right)$ due to shearing can be calculated:

$E_{V}=\frac{P \cdot t}{V}$

\subsection{Sediment compression analysis}

Sediment compression of the samples was measured with the LUMiSizer. The cuvettes were filled with approximately $2 \mathrm{ml}$ undiluted sample. LUMiSizer speed was varied between $1500 \mathrm{rpm}$ and $4000 \mathrm{rpm}$ in steps of $500 \mathrm{rpm}$. As the maximum sedimentation velocity of the cultures is a few hundred $\mu \mathrm{m} / \mathrm{s}$ only, the measurements were run at least overnight and at $1500 \mathrm{rpm}$ for up to $20 \mathrm{~h}(+30 \mathrm{~min}$ for each 


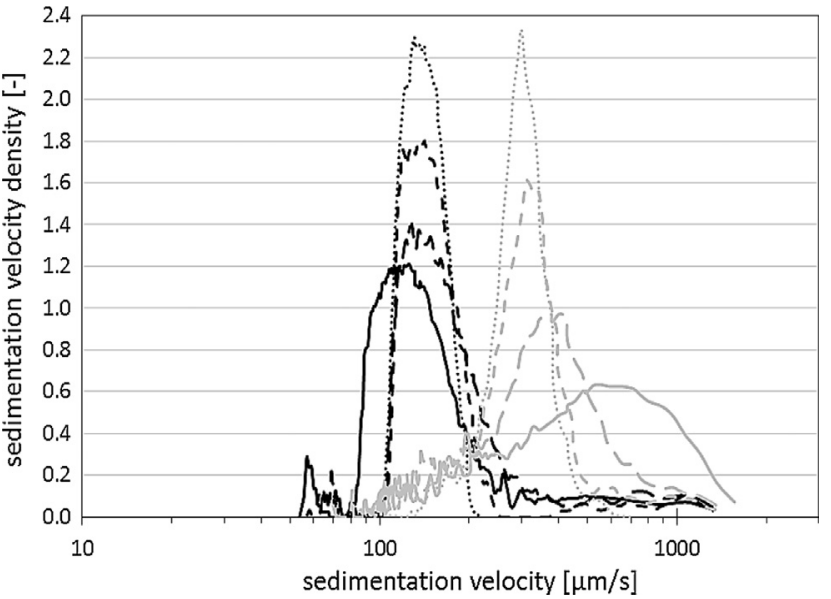

Fig. 1. Sedimentation velocity distributions of the unsheared (full line) bacteria strains ST-D (free EPS, gray) and ST-C (capsular EPS, black) and after shearing at 19,000 rpm for $10 \mathrm{~s}$ (long dashed line), $30 \mathrm{~s}$ (short dashed line), and $120 \mathrm{~s}$ (dotted line).

higher rpm). Sedimentation of the samples was considered to be stable when the instability index of the LUMiSizer measurement was constant. The sediment height for each sample was calculated from the difference between the radial position at a transmission of $50 \%$ and the experi mentally determined cell bottom (by measuring empty cuvettes and cuvettes filled with water).

\section{Results and discussion}

\subsection{Sedimentation velocity analyses}

Fig. 1 shows the sedimentation velocity distribution plots from LUMiSizer analyses of the capsular EPS producing strain ST C and the free EPS producing strain ST D after UT shear treatment at 19,000 rpm for different times. The unsheared ST D exhibits a very broad sedi mentation velocity distribution, from 100 to almost $2000 \mu \mathrm{m} / \mathrm{s}$. This can be attributed to the ability of the bacteria to form long cell chains (2 19 cocci per chain, see Table 1), resulting in increasing volume of particulate objects and apparent particle size. Due to the broad dis tribution, the velocity distribution density, which is an indicator of the amount of particles that sediment at the same speed, is very low. Ten seconds of shearing with a UT speed of $19,000 \mathrm{rpm}$ decreased the amount of fast sedimenting particles and increased the amount of slower sedimenting particles. As a consequence, the maximum velocity distribution density increased from 0.6 (unsheared) to 1.0 (sheared). This reflects partial disruption of cell chains due to shearing (Fig. 2). With increasing shear duration, cell chains were disrupted to a larger extent, which led to a further decrease of sedimentation velocity. After $120 \mathrm{~s}$ of shearing at $19,000 \mathrm{rpm}$, the mean chain length decreased from 13 to 6 cells and a single peak was observed with a maximum velocity distribution density of 2.4 at a sedimentation velocity of $300 \mu \mathrm{m} / \mathrm{s}$.

In contrast to ST D, shearing of the capsular EPS producing strain ST C leads to higher sedimentation velocities. The unsheared sample showed a main sedimentation velocity peak from 80 to $250 \mu \mathrm{m} / \mathrm{s}$ with a maximum sedimentation velocity distribution density of 1.4 at a sedi mentation velocity of approximately $120 \mu \mathrm{m} / \mathrm{s}$. Faster sedimenting particles (presumably cell chains) and slower sedimenting particles (presumably cell fragments or fines) are also visible. After shearing for $10 \mathrm{~s}$ at a UT speed of $19,000 \mathrm{rpm}$, a shift of the sedimentation velocity distribution to higher sedimentation velocities is obvious. This shift can be explained by inspecting the capsular EPS layer that is produced by ST C (see Fig. 2 for microscope images of the unsheared and sheared cultures). The sample was mixed with India ink and, as the ink particles cannot permeate into the polysaccharide, the capsular EPS layer is visible as a bright layer around the cells $[14,15]$. The microscope images indicate that the capsules were removed during shearing. Due to the high water binding capacity of the EPS, we assume that the capsular EPS layer around the cells acts as a kind of friction pad and reduces the sedimentation velocity of the cells. Hence, a shift to a higher sedi mentation velocity is the expected consequence after shearing off the capsular EPS layer. With increasing shear duration, no further shift to a higher sedimentation velocity occurred, while the disruption of the cell chains was clearly visible. After $120 \mathrm{~s}$ of shearing, the maximum sedi mentation velocity distribution density increased to 2.3 at a sedi mentation velocity of approximately $140 \mu \mathrm{m} / \mathrm{s}$. This indicates that the capsular EPS are sheared off prior to a disruption of the cell chains. Similar results, i.e. an increase in sedimentation velocity due to shearing, were observed for the other free EPS producing strain (ST E). In this particular case, the increasing sedimentation velocity can be attributed to changes of the viscosity of the surrounding fermentation broth. After shearing the medium for $120 \mathrm{~s}$ at $19,000 \mathrm{rpm}$, the apparent viscosity of the cell free broth decreased by $40 \%$. Hence, the mean sedimentation velocity increase from 182 to $206 \mu \mathrm{m} / \mathrm{s}$ can be explained by Stokes' law.

The sedimentation velocity of the bacteria was also measured with a disc centrifuge to facilitate the comparison of the results with the LUMiSizer. Table 2 shows the influence of the shear treatment on se dimentation velocities of the strains ST C, ST D, and ST E. In addition to the median value for the sedimentation velocities (v50\%), the v10\% and $\mathrm{v} 90 \%$ values are listed, as they are important for interpreting the shearing effects. This table clearly illustrates the interactions between the three effects on sedimentation velocity caused by the shear treat ment. From the LUMiSizer data for ST C, both the increase in sedi mentation velocity due to shearing off capsular EPS (v10\%) and the decrease due to disruption of cell chains (v90\%) is visible. Looking at the average sedimentation velocity, it becomes obvious that it is af fected by both (increase and then decrease with increasing shearing time). The disc centrifuge only shows increasing values for ST C, which can be explained by the high shear force jump that occurs as soon as the particles penetrate the fast spinning density gradient. The few longer cell chains are disrupted and no difference between sheared and un sheared samples can be determined. In the LUMiSizer the cell chains are accelerated together with the surrounding medium and therefore no shear stress jump occurs and consequently the cell chains are not dis rupted. LUMiSizer measurements of ST E showed increasing sedi mentation values, but no significant changes in the disc centrifuge, which reflects the observed change in medium viscosity. In the LUMi Sizer a decreasing medium viscosity results in an increase in sedi mentation velocity. Hence, the particles are sedimenting through the medium. In the disc centrifuge the particles are sedimenting through a density gradient, whose viscosity remains unaffected during shearing of the samples. The slightly decreasing v90\% values measured with the disc centrifuge are a consequence of disrupting shorter cell chains. In almost all cases, ST D showed a decreasing sedimentation velocity caused by disrupting cell chains. Only the v10\% values measured with the LUMiSizer were found to increase, which is a consequence of the decreasing medium viscosity similar to ST E.

\subsection{Influence of energy input on sedimentation velocity}

Fig. 3 illustrates that there are large differences in the sedimentation velocity of the six ST strains and shear treatment affected sedimentation velocity to a different extent. The fastest sedimenting strain is ST D which produced free EPS and formed the longest cell chains (see Table 1). The other free EPS producing strain ST E exhibited a sig nificantly lower sedimentation velocity than ST D, which, in turn, was higher than that of the four capsular EPS producing strains. This is another indicator which allows to interpret the capsular EPS layers as decelerating friction pads. For all strains, the sedimentation velocity showed a logarithmic dependency on the energy input or stayed nearly constant. The tendency of the capsular EPS producing strains to show an increasing sedimentation velocity with increasing energy input can 


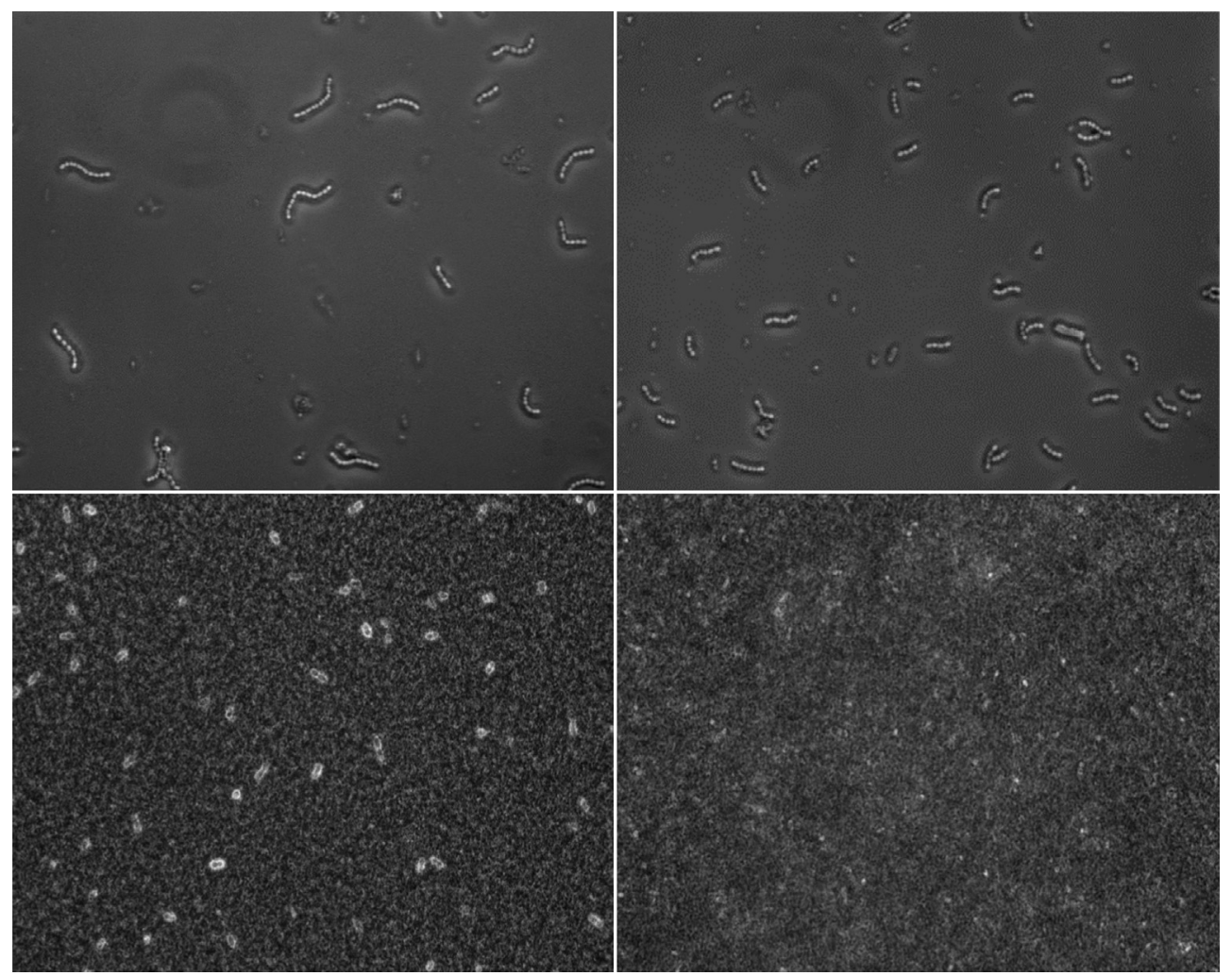

Fig. 2. Microscopies of the free EPS-producing strain ST-D unsheared (top left) and sheared for $120 \mathrm{~s}$ at 24,000 rpm (top right). Microscopies with India ink of the capsular EPS-producing strain ST-C unsheared (bottom left) and sheared at 24,000 rpm for $120 \mathrm{~s}$ (bottom right).

be explained by shearing off the decelerating EPS layer around the cells. The cell chain disruption observed for all strains leads to two opposite effects on sedimentation velocity. This might be a possible explanation why the sedimentation velocity values of ST C (LUMiSizer) deviate pronouncedly from the logarithmic fit.

\subsection{Influence of energy input intensity}

Fig. 4 shows the sedimentation velocity distribution densities of the unsheared ST C and of ST C that was sheared for $120 \mathrm{~s}$ at different UT speeds. It is obvious that the cell chains were almost completely dis rupted after $120 \mathrm{~s}$ of shearing irrespective of the shear speed applied. As regards the position of the maximum peak height, it was found that a higher energy input intensity and, hence, a higher total energy input led to increasing sedimentation velocities.
Fig. 5 shows the sedimentation velocity distributions of ST C and ST D sheared at different shear speeds for variable shear durations, but at a similar energy input. An energy input of $12.5 \mathrm{~J} / \mathrm{ml}$ was achieved by shearing at $11,000 \mathrm{rpm}$ for $10 \mathrm{~s}$ or by shearing at $5000 \mathrm{rpm}$ for $60 \mathrm{~s}$. The corresponding values for high and low shear intensities at an energy input of $1050 \mathrm{~J} / \mathrm{ml}$ were $24,000 \mathrm{rpm}$ for $60 \mathrm{~s}$ or $19,000 \mathrm{rpm}$ for $120 \mathrm{~s}$, respectively. At similar energy inputs, the sedimentation velocity dis tribution significantly depends on the conditions under which this en ergy was achieved. At $12.5 \mathrm{~J} / \mathrm{ml}$ for ST C, the lower shear duration at higher shear speed resulted in a higher sedimentation velocity and in a broader distribution range than the higher shear duration at lower shear speed. This indicates that the capsular EPS were sheared off more effectively at higher shear speed, while most of the cell chains remained unaffected. Similar effects were observed for ST D, indicating that cell chain disruption at higher shearing intensity is lower. Data obtained at

\section{Table 2}

Sedimentation velocities of ST-C, ST-D, and ST-E as a function of shear duration at 19,000 rpm, measured with the disc centrifuge and the LUMiSizer. The table is normalized to the sedimentation velocities of the unsheared samples.

\begin{tabular}{|c|c|c|c|c|c|c|c|}
\hline \multirow[t]{3}{*}{ Strain } & \multirow{3}{*}{$\begin{array}{l}\text { Shear } \\
\text { duration }[s]\end{array}$} & \multicolumn{3}{|c|}{ LUMiSizer } & \multicolumn{3}{|c|}{ Disc centrifuge } \\
\hline & & \multicolumn{3}{|c|}{ Sedimentation velocity $[\mu \mathrm{m} / \mathrm{s}]$} & \multicolumn{3}{|c|}{ Sedimentation velocity $[\mu \mathrm{m} / \mathrm{s}]$} \\
\hline & & $\mathrm{v} 10 \%$ & v50\% & v90\% & v10\% & v50\% & v90\% \\
\hline \multirow[t]{4}{*}{ ST-C } & 0 & 100 & 100 & 100 & 100 & 100 & 100 \\
\hline & 10 & 126 & 104 & 121 & 118 & 114 & 109 \\
\hline & 30 & 126 & 112 & 59 & 122 & 118 & 112 \\
\hline & 120 & 124 & 103 & 49 & 131 & 126 & 118 \\
\hline \multirow[t]{4}{*}{ ST-D } & 0 & 100 & 100 & 100 & 100 & 100 & 100 \\
\hline & 10 & 93 & 72 & 73 & 93 & 92 & 91 \\
\hline & 30 & 108 & 64 & 47 & 96 & 96 & 95 \\
\hline & 120 & 124 & 60 & 36 & 93 & 91 & 89 \\
\hline \multirow[t]{4}{*}{ ST-E } & 0 & 100 & 100 & 100 & 100 & 100 & 100 \\
\hline & 10 & 116 & 111 & 117 & 100 & 100 & 98 \\
\hline & 30 & 112 & 112 & 105 & 100 & 99 & 92 \\
\hline & 120 & 112 & 113 & 110 & 101 & 99 & 92 \\
\hline
\end{tabular}




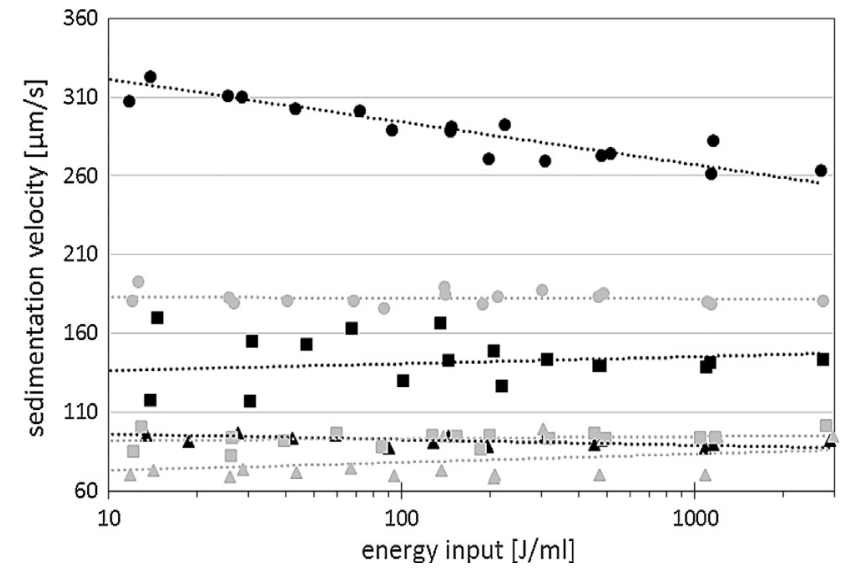

Fig. 3. Influence of shear energy input on sedimentation velocity of bacteria cells, measured with the LUMiSizer. Strain identifiers: ST-C, black square; ST-D, black circle; ST-E, gray circle; ST-G, black triangle; ST-H, gray triangle; ST-I, gray square. (For interpretation of the references to colour in this figure legend, the reader is referred to the web version of this article.)

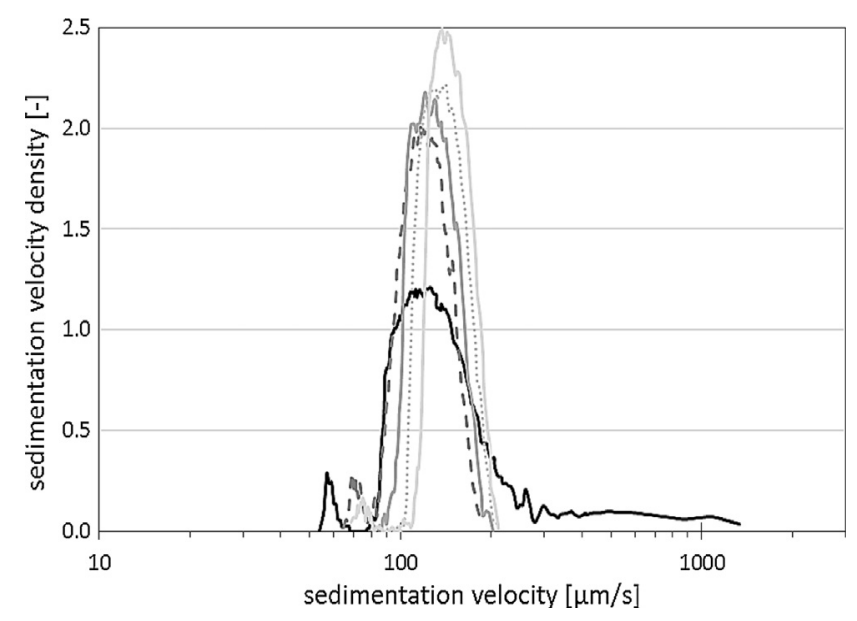

Fig. 4. Influence of shear energy on the sedimentation velocity distribution of the capsular EPS producer ST-C. Black is the unsheared reference and the shear duration for all sheared samples was $120 \mathrm{~s}$. Shear speed was set to $5000 \mathrm{rpm}$ (dashed gray line), 11,000 rpm (solid gray line), 19,000 rpm (dotted light gray line), or 24,000 rpm (solid light gray line). (For interpretation of the references to colour in this figure legend, the reader is referred to the web version of this article.)

an energy input of $190 \mathrm{~J} / \mathrm{ml}$ confirmed this (data not shown). At an energy input of $1050 \mathrm{~J} / \mathrm{ml}$, however, shear intensity only slightly af fected the sedimentation distribution. Single peaks suggest that all cell chains were disrupted already. This means that both the capsular EPS producing and the free EPS producing cultures exhibited smaller cell chain disruption and, consequently, higher mean sedimentation velo cities when sheared with high intensity for a short duration.

\subsection{Sediment compression}

The influence of shear treatment on the cell sediments of the dif ferent bacteria strains was investigated as well. Fig. 6 illustrates the sedimentation kinetics of the capsular EPS producing ST C, either un sheared or sheared at $19,000 \mathrm{rpm}$ for different times. The sharp bend of the gradient reflects the transition from sedimentation (high gradient) to sediment compression (low gradient). The incline that can be seen in all curves after sedimentation indicates that the sediment is compres sible irrespective of a preceding shearing procedure. It is obvious that with longer shear treatment the point of transition was reached earlier. Sediment height before compression decreased (a higher radial position indicates a lower sediment height). This also means that a higher shear treatment led to a faster sedimentation and a more pronounced se paration before sediment compression started. The sedimentation
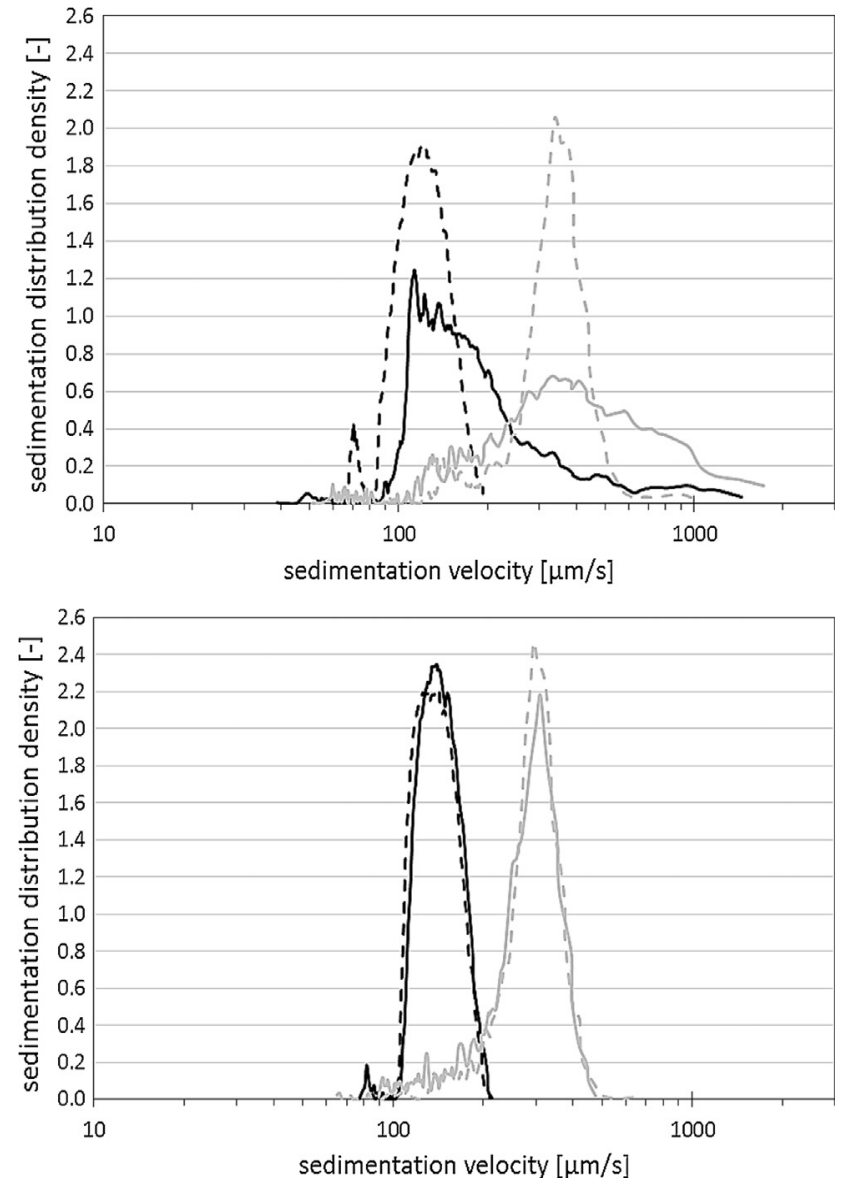

Fig. 5. Comparison of the influence of different shear intensities at similar energy input (upper chart, $12.5 \mathrm{~J} / \mathrm{ml}$; lower chart, $1050 \mathrm{~J} / \mathrm{ml}$ ) on the sedimentation velocity distribution of the capsular EPS-producing ST-C (black) and the free EPS-producing ST-D (gray). The solid lines show the higher shear intensity (higher UT speed), the dashed lines the lower shear intensity at the same energy input (higher shear duration). (For interpretation of the references to colour in this figure legend, the reader is referred to the web version of this article.)

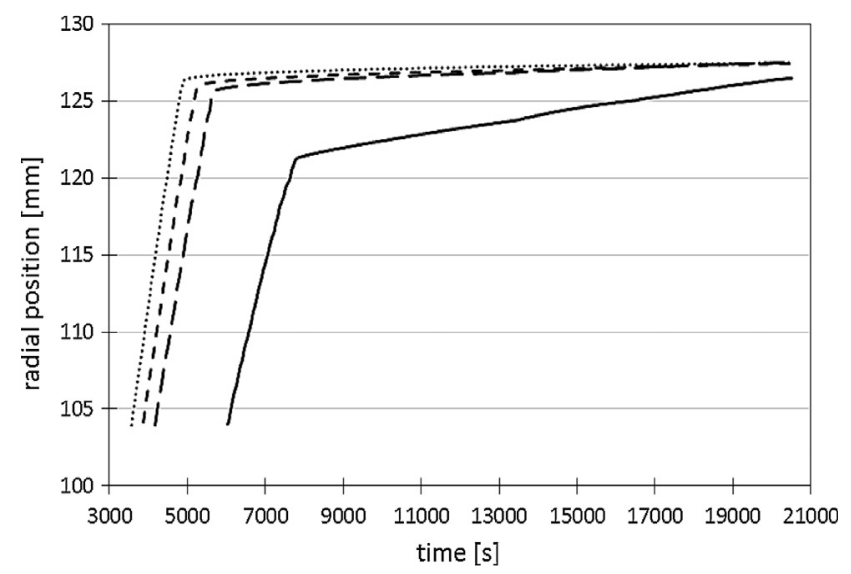

Fig. 6. Influence of shear treatment on sedimentation kinetics of the capsular EPS-producing ST-C. Shearing was at 19,000 rpm for $0 \mathrm{~s}$ (solid line), $10 \mathrm{~s}$ (long dashed line), $30 \mathrm{~s}$ (short dashed line), or $120 \mathrm{~s}$ (dotted line).

kinetics of the unsheared sample indicates that there are some inter actions between the particles (e.g. rubbing or getting stuck with each other) and that these interactions are weakened through shearing [16,17]. Shearing off the capsular EPS layer might be the reason for those changing interdependencies between the particles, as shearing led to faster sedimentation velocities, but also to more compact sediments. Both are desired in the production of starter cultures. A similar, but weaker effect was observed for the free EPS producing strain ST D (data not shown). Here, an increase in sediment compactness can be 


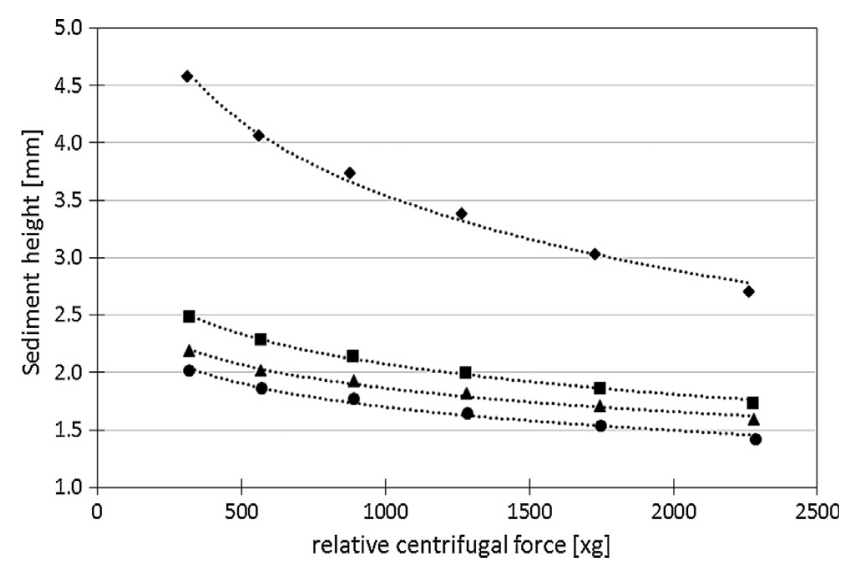

Fig. 7. Influence of shear treatment and relative centrifugal force on the sediment height of a capsular EPS-producing strain. Shearing was at 19,000 rpm for $0 \mathrm{~s}$ (rhomb), $10 \mathrm{~s}$ (square), $30 \mathrm{~s}$ (triangle), or $120 \mathrm{~s}$ (circle).

attributed to the disruption of cell chains and sedimentation is faster because of decreasing viscosity. Only the free EPS producing strain ST E showed no compressive sediment and remained unaffected by shearing in terms of sediment height (data not shown). Similar to ST D, however, the reduced medium viscosity led to faster sedimentation velocities. Consequently, the final sediment height was reached earlier.

Fig. 7 exemplarily shows the influence of shearing and rcf on the sediment height of a capsular EPS producing strain (ST I). With in creasing rcf, the sediment height decreases logarithmically due to the higher forces acting on the sediment. This indicates that the sediment of this strain is compressible. With increasing shear forces, sediment height also decreased so that shearing led to more compact sediments. This effect is the consequence of shearing off capsular EPS. This reduces the interdependencies between particles and causes more compact se diments. This behavior is also visible for the capsular EPS producing ST C and, in an attenuated form, for the long cell chains and free EPS producing ST D. The other free EPS producing ST E strain showed no significant changes in sediment height and, hence, was affected neither by shearing nor by increasing the rcf.

\section{Conclusion}

In this study it is shown that the sedimentation velocity of bacteria cells in fermentation media can be affected by shearing. The following effects were observed: (a) the strongest positive effect was an increased sedimentation velocity caused by shearing off capsular EPS; (b) for strains producing free EPS only, the sedimentation velocity was in creased because shearing reduced the apparent fermentation broth viscosity; and (c) the disruption of long cell chains resulted in a smaller particle size and, consequently, a lower sedimentation velocity. Comparison of the mean sedimentation velocities revealed a combina tion of all three effects. The change in viscosity led to faster sediment building because of the higher sedimentation velocity, but had no effect on sediment compression. The disruption of cell chains reduced sedimentation velocity, but resulted in a more compact sediment thanks to particle size decrease. Shearing off capsular EPS from the cells re duced the interdependencies between the particles and, hence, resulted in a strong compaction of the sediment, which may facilitate the se paration of the cells in the course of starter culture production.

\section{Acknowledgements}

We would like to thank all the companies involved in our project committee for their support of this study. Special thanks go to Chr. Hansen for providing the fermentation media with different bacteria strains. We thank the AiF for their financial support.

This IGF Project 18605 BG of the FEI is supported via AiF within the programme for promoting the Industrial Collective Research (IGF) of the German Ministry of Eco nomics Affairs and Energy (BMWi), based on a resolution of the German Parliament.

\section{References}

[1] D. Jaros, H. Rohm, The rheology and textural properties of yoghurt, in B.M. McKenna (Ed.), Texture of Foods, Woodhead, Cambridge, 2003, pp. 321-349.

[2] L. Jolly, J.F. Vincent, P. Duboc, J. Neeser, Exploiting Exopolysaccharides from Lactic Acid Bacteria, Antonie Van Leeuwenhoek, 2002, pp. 367-374.

[3] L. De Vuyst, F. de Vin, F. Vaningelgem, B. Degeest, Recent developments in the biosynthesis and applications of heteropolysaccharides from lactic acid bacteria, Int. Dairy J. 11 (2001) 687-707.

[4] F. Vaningelgem, M. Zamfir, F. Mozzi, T. Adriany, M. Vancanneyt, J. Swings, et al., Biodiversity of exopolysaccharides produced by Streptococcus thermophilus strains is reflected in their production and their molecular and functional characteristics, Appl. Environ. Microbiol. 70 (2004) 900e912.

[5] A.S. Kumar, K. Mody, Microbial exopolysaccharides: variety and potential applications, in: Microb. Prod. Biopolym. Polym. Precursors Appl. Perspect., Rehm, Bern, Caister Academic Press, 2009.

[6] S. Mende, M. Peter, K. Bartels, H. Rohm, D. Jaros, Food hydrocolloids: addition of purified exopolysaccharide isolates from S. thermophilus to milk and their impact on the rheology of acid gels, Food Hydrocolloids 32 (2013) 178-185.

[7] T. Detloff, D. Lerche, Centrifugal separation in tube and disc geometries: experiments and theoretical models, Acta Mech. 201 (2008) 83-94.

[8] D. Lerche, Dispersion stability and particle characterization by sedimentation kinetics in a centrifugal field, J. Dispers. Sci. Technol. 23 (2002) 699-709.

[9] T. Detloff, T. Sobisch, D. Lerche, Particle Size distribution by space or time dependent extinction profiles obtained by analytical centrifugation (concentrated systems), Powder Technol. 174 (2007) 50-55.

[10] David J. Scott, Stephen E. Harding, Arthur J. Rowe, Analytical ultracentrifugation: techniques and methods, Chapter 14, Introduction to Differential Sedimentation, I. Laidlaw, M. Steinmetz, 2005.

[11] M. Zlokarnik, Rührtechnik, Springer, Theorie und Praxis, 1999.

[12] S. Weiß (Ed.), Verfahrenstechnische Berechnungsmethoden. Stoffvereinigen in fluiden Phasen, Rührausrüstungen und ihre Berechnung. Deutscher Verlag für Grundstoffindustrie, 1988.

[13] A.T. Utomo, M. Baker, A.W. Pacek, Flow pattern, periodicity and energy dissipation in a batch rotor-stator mixer, Chem. Eng. Res. Des. 86 (2008) 1397-1409.

[14] N.E. Costa, L. Wang, M.E. Auty, J.A. Hannon, et al., Rheological, microscopic and primary chemical characterisation of the exopolysaccharide produced by Lactococcus lactis susp. cremoris DPC6532, Dairy Sci. Technol. 92 (2012) 219-235.

[15] N.A. Yurlova, G.S. de Hoog, Exopolysaccharides and capsules in human pathogenic Exophiala species, Mycoses 45 (2002) 11-12.

[16] T. Sobisch, D. Lerche, Charakterisierung interpartikulärer Kräfte für Fest/FlüssigTrennverfahren mittels analytischer Zentrifugation, Chem. Ing. Tech. 80 (2008) 393-397.

[17] D. Lerche, T. Sobisch, Evaluation of particle interactions by in situ visualization of separation behavior, Colloids Surf. A 440 (2014) 122-130. 


\section{Repository KITopen}

Dies ist ein Postprint/begutachtetes Manuskript.

Empfohlene Zitierung:

Häffele, F.; Mende, S.; Jaros, D.; Rohm, H.; Nirschl, H.

Relationship between shear energy input and sedimentation properties of exopolysaccharide-producing Streptococcus thermophilus strains. 2018. Separation and purification technology, 202.

doi: $10.5445 / / R / 1000081481$

Zitierung der Originalveröffentlichung:

Häffele, F.; Mende, S.; Jaros, D.; Rohm, H.; Nirschl, H.

Relationship between shear energy input and sedimentation properties of exopolysaccharide-producing Streptococcus thermophilus strains.

2018. Separation and purification technology, 202, 21-26.

doi:10.1016/j.seppur.2018.03.031 